\title{
Fuzzy Transportation Linear Programming Models based on L-R Fuzzy Numbers
}

\author{
Y.L.P.Thorani \\ Dept.of Applied Mathematics \\ GIS, GITAM University \\ Visakhapatnam, India
}

\author{
N.Ravi Shankar \\ Dept.of Applied Mathematics \\ GIS, GITAM University \\ Visakhapatnam, India
}

\begin{abstract}
Transportation models play an important role in logistics and supply chain management for reducing cost and improving service. In this paper two new fuzzy transportation linear programming models are developed: one with equality constraints and other with inequality constraints using L-R fuzzy numbers. The membership functions of $L-R$ fuzzy numbers of fuzzy transportation cost are consider being linear and exponential. This paper develops a procedure to derive the fuzzy objective value of the fuzzy transportation problem, in that the cost coefficients and the supply and demand are L-R fuzzy numbers. The two models are illustrated with an example. The optimal fuzzy transportation cost for the two models slightly varies when linear membership functions are equal and the optimal fuzzy transportation cost is same in case of different membership functions i.e., either linear or exponential membership functions defined on L-R fuzzy numbers. Most of the fuzzy transportation problems reviewed in literature have the negative optimal fuzzy transportation cost but in our proposed method we obtain positive optimal fuzzy transportation cost in all most all cases.
\end{abstract}

\section{Keywords}

Fuzzy transportation problem; Yager's ranking index; L-R fuzzy numbers; linear programming.

\section{INTRODUCTION}

In today's highly competitive market the pressure on organizations to find better ways to create and deliver value to customers becomes stronger. To send the products to the customers in the quantities the organizers want in a costeffective manner become more challenging. Transportation models provide a powerful frame work to meet this challenge. The organizers ensure the efficient movement and timely availability of raw materials and finished goods. The application of the transportation problem is not limited to transporting commodities between sources and destinations. The application of transportation problem was first posed by Ferguson and Dantiz [1] in a paper discussing the allocation of aircraft to routes. Many research papers [2-4] are introduced for solving such type of transportation problems. A dual method for solving transportation problem was presented by Balas [11] and an operator theory of parametric programming for solving transportation problem was presented by Balachandran and Thompson [5-7]. The works referred so far for solving the transportation problem assume that the parameters of the problem are exactly known. However, there are situations when the parameters may not be known in a precise manner. To deal with such situations, fuzzy set theory has been applied in literature to solve the transportation problem and to enrich and enhance the suggested solution methodologies. Bit et al. [12] consider a kobjective transportation problem fuzzified by fuzzy numbers and used $\alpha$-cut to obtain a transportation problem in the fuzzy sense expressed in linear programming form. Chanas and Kuchta [13] used fuzzy numbers of the type L-L to fuzzify cost coefficients in the objective function and $\alpha$-cut to express the objective function in the form of an interval. Hussien [14] studied the complete set of $\alpha$-possibility efficient solutions of multi objective transportation problem with possibilistic co-efficients of the objective functions. Li and Lai [15] proposed a fuzzy compromise programming approach to a multi objective linear transportation problem. Zimmermann [16] showed that solutions obtained by fuzzy linear programming are always efficient. Subsequently, Zimmermann's fuzzy linear programming has developed into several fuzzy optimization methods for solving the transportation problems. Oheigeartaigh [17] proposed an algorithm for solving transportation problems where the capacities and requirements are fuzzy sets with linear or triangular membership functions. Chanas et al. [18] presented a fuzzy linear programming model for solving transportation problems with crisp cost coefficients and fuzzy supply and demand values. Chanas and Kuchta [19] proposed the concept of the optimal solution for the transportation problem with fuzzy coefficients expressed as fuzzy numbers and developed an algorithm for obtaining the optimal solution. Saad and Abbas [20] discussed the solution algorithm for solving the transportation problem in fuzzy environment. Liu and Kao [21] described a method for solving fuzzy transportation problems based on extension principle. Kaur and Kumar [24] proposed a method for solving fuzzy transportation problems by assuming that a decision maker is uncertain about the precise values of the transportation cost, availability and demand of the product are represented by generalized trapezoidal fuzzy numbers and illustrated with an example. Most of the fuzzy transportation problems discussed above, the optimal fuzzy transportation cost is negative in nature but in our proposed method we obtain positive optimal fuzzy transportation cost in all most all cases.

The rest of the paper is organized as follows. In section 2 preliminaries of L-R fuzzy numbers, $\lambda$-cut of L-R fuzzy number, reference functions and Yager's ranking approach for the fuzzy numbers are presented. In section 3 the proposed Linear programming models for fuzzy transportation problem has been given. In section 4 the proposed method to find the optimal solution for fuzzy transportation models has been given. In section 5 we discuss the application of proposed models with a numerical example using various cases and the comparison of Optimal fuzzy transportation cost of the two models are given. Finally the conclusion is given in section 6 . 


\section{PRELIMINARIES}

In this section, L-R fuzzy numbers, $\lambda$-cut of L-R fuzzy number, reference functions and Yager's ranking approach for the fuzzy numbers are presented.

\subsection{L-R fuzzy numbers and reference functions}

In this section, L-R fuzzy number, $\lambda$-cut of L-R fuzzy number, and reference functions are reviewed [22].

Definition 1 A fuzzy number $\tilde{\mathrm{A}}=(\mathrm{m}, \mathrm{n}, \alpha, \beta)_{\mathrm{L}-\mathrm{R}}$ is said to be an L-R fuzzy number if

$$
\mu_{\tilde{A}}(x)=\left\{\begin{array}{lc}
L\left(\frac{m-x}{\alpha}\right), & x \leq m, \alpha>0, \\
R\left(\frac{x-n}{\beta}\right), & x \geq n, \beta>0, \\
1, & \text { otherwise. }
\end{array}\right.
$$

If $\mathrm{m}=\mathrm{n}$ then $\tilde{\mathrm{A}}=(\mathrm{m}, \mathrm{n}, \alpha, \beta)_{\mathrm{L}-\mathrm{R}} \quad$ will be converted into $\tilde{\mathrm{A}}=(\mathrm{m}, \alpha, \beta)_{\mathrm{L}-\mathrm{R}}$ and is said to be an L-R fuzzy number.

$\mathrm{L}$ and $\mathrm{R}$ are called reference functions, which are continuous, non-increasing functions that define the left and right shapes of $\mu_{\tilde{\mathrm{A}}}(\mathrm{x})$ respectively and $\mathrm{L}(0)=\mathrm{R}(0)=1$. Two special cases are triangular and trapezoidal fuzzy number, for which $\mathrm{L}(\mathrm{x})=\mathrm{R}(\mathrm{x})=\max \{0,1-|\mathrm{x}|\}$ are linear functions.

Nonlinear reference functions which are commonly used with parameters $\mathrm{p}$, denoted as $\mathrm{RF}_{\mathrm{p}}(\mathrm{x})$. Linear and Non-linear reference functions with their inverses are presented in Table I.

Definition 2 Let $\tilde{\mathrm{A}}=(\mathrm{m}, \mathrm{n}, \alpha, \beta)_{\mathrm{L}-\mathrm{R}}$ be an L-R fuzzy number and $\lambda$ be a real number in the interval $[0,1]$. Then the crisp set $\mathrm{A}_{\lambda}=\left\{\mathrm{x} \in \mathrm{X}: \mu_{\tilde{\mathrm{A}}}(\mathrm{x}) \geq \lambda\right\}=\left[\mathrm{m}-\alpha \mathrm{L}^{-1}(\lambda), \mathrm{n}+\beta \mathrm{R}^{-1}(\lambda)\right]$ is said to be $\lambda$-cut of $\tilde{\mathrm{A}}$.

\subsection{Yager's Ranking for L-R fuzzy numbers} In this section, Yager's method [23] is presented for the ranking of L-R fuzzy numbers. This method involves a procedure for ordering fuzzy sets in which a ranking approach $\mathrm{R}(\tilde{\mathrm{A}})$ is calculated for the fuzzy number $\tilde{\mathrm{A}}=(\mathrm{m}, \mathrm{n}, \alpha, \beta)_{\mathrm{L}-\mathrm{R}}$ from its $\lambda$-cut $A_{\lambda}=\left[m-\alpha L^{-1}(\lambda), n+\beta R^{-1}(\lambda)\right]$ according to the following formula:

$$
\mathrm{R}(\tilde{\mathrm{A}})=\frac{1}{2}\left(\int_{0}^{1}\left(\mathrm{~m}-\alpha \mathrm{L}^{-1}(\lambda)\right) \mathrm{d} \lambda+\int_{0}^{1}\left(\mathrm{n}+\beta \mathrm{R}^{-1}(\lambda)\right) \mathrm{d} \lambda\right)
$$

Let $\tilde{\mathrm{A}}$ and $\tilde{\mathrm{B}}$ be two fuzzy numbers. Then

(i) $\tilde{\mathrm{A}}>\tilde{\mathrm{B}}$ if $\mathrm{R}(\tilde{\mathrm{A}})>\mathrm{R}(\tilde{\mathrm{B}})$

(ii) $\tilde{\mathrm{A}}=\tilde{\mathrm{B}}$ if $\mathrm{R}(\tilde{\mathrm{A}})=\mathrm{R}(\tilde{\mathrm{B}})$

(iii) $\tilde{\mathrm{A}}<\tilde{\mathrm{B}}$ if $\mathrm{R}(\tilde{\mathrm{A}})<\mathrm{R}(\tilde{\mathrm{B}})$

\section{PROPOSED LINEAR} PROGRAMMING MODELS FOR FUZZY TRANSPORTATION PROBLEM

Generally the fuzzy transportation problem is to transport various amounts of a single homogeneous commodity that are initially stored at various sources, to different destinations in such a way that the total fuzzy transportation cost is a minimum. Let there be $m$ sources, $i^{\text {th }}$ sources possessing $\tilde{a}_{i}$ fuzzy supply units of a certain product, $\mathrm{n}$ destinations ( $\mathrm{n}$ may or may not be equal to $m$ ) with destination $j$ requiring $\tilde{b}_{j}$ fuzzy demand units. Cost of shipping of an item from each of $m$ sources to each of the $n$ destinations are known either directly or indirectly in terms of mileage, shipping hours, etc. Let $\tilde{c}_{\mathrm{ij}}$ be the fuzzy cost of shipping one unit product from $\mathrm{i}^{\text {th }}$ source to $\mathrm{j}^{\text {th }}$ destination, and $\mathrm{x}_{\mathrm{ij}}$ be the amount to be shipped from $i^{\text {th }}$ sources to $j^{\text {th }}$ destination. Fuzzy transportation problem with fuzzy cost $\tilde{c}_{\mathrm{ij}}$, fuzzy supply units $\tilde{\mathrm{a}}_{\mathrm{i}}$ and fuzzy demand units $\tilde{b}_{j}$ is given in Table II.

\subsection{Proposed Model for fuzzy transportation problem with equality constraints}

Fuzzy Approach to the transportation problem based on equality condition mathematically is defined as follows:

Minimize $\tilde{\mathrm{c}}=\sum_{\mathrm{i}=1}^{\mathrm{m}} \sum_{\mathrm{j}=1}^{\mathrm{n}} \tilde{\mathrm{c}}_{\mathrm{ij}} \mathrm{x}_{\mathrm{ij}}$

subject to constraints

$$
\begin{aligned}
& \sum_{j=1}^{n} x_{i j} \cong \tilde{a}_{i} \quad i=1,2, \ldots, m \\
& \sum_{i=1}^{m} x_{i j} \cong \tilde{b}_{j} \quad j=1,2, \ldots, n \\
& x_{i j} \geq 0 \quad i=1,2, \ldots, m ; j=1,2, \ldots, n .
\end{aligned}
$$

where $\tilde{\mathrm{c}}_{\mathrm{ij}}=\left(\mathrm{m}_{\mathrm{ij}}, \mathrm{n}_{\mathrm{ij}}, \alpha_{\mathrm{ij}}, \beta_{\mathrm{ij}}\right)_{\mathrm{L}-\mathrm{R}}:$ Fuzzy payment from $\mathrm{i}^{\text {th }}$ source to $\mathrm{j}^{\text {th }}$ destination

$$
\begin{aligned}
& \mathrm{L}(\mathrm{x})=\mathrm{R}(\mathrm{x})=\max \{0,1-|\mathrm{x}|\} \quad \text { or } \quad \mathrm{L}(\mathrm{x})=\mathrm{R}(\mathrm{x})=\mathrm{e}^{-\mathrm{x}} \text { or } \\
& \mathrm{L}(\mathrm{x})=\max \{0,1-|\mathrm{x}|\} \text { and } \mathrm{R}(\mathrm{x})=\mathrm{e}^{-\mathrm{x}} \text { or } \\
& \mathrm{L}(\mathrm{x})=\mathrm{e}^{-\mathrm{x}} \text { and } \mathrm{R}(\mathrm{x})=\max \{0,1-|\mathrm{x}|\} \\
& \sum_{\mathrm{i}=1}^{\mathrm{m}} \sum_{\mathrm{j}=1}^{\mathrm{n}} \tilde{\mathrm{c}}_{\mathrm{ij}} \mathrm{x}_{\mathrm{ij}}: \text { Total fuzzy cost from } \mathrm{i}^{\text {th }} \text { source to } \mathrm{j}^{\text {th }} \\
& \text { destination. }
\end{aligned}
$$

where $\tilde{\mathrm{a}}_{\mathrm{i}}=$ fuzzy quantity of supply $\mathrm{i}$,

$\tilde{b}_{j}=$ fuzzy quantity of demand $j$,

$\tilde{c}_{i j}=$ the fuzzy cost from $i^{\text {th }}$ source to $j^{\text {th }}$ destination.

$\mathrm{L}_{\mathrm{ij}}(\mathrm{x})=$ left shape functions,

$\mathrm{R}_{\mathrm{ij}}(\mathrm{x})=$ right shape functions.

All $\tilde{a}_{\mathrm{i}}, \tilde{\mathrm{b}}_{\mathrm{j}}, \tilde{\mathrm{c}}_{\mathrm{ij}}$ denotes a non-negative L-R fuzzy numbers. 


\subsection{Proposed Model for fuzzy transportation problem with inequality constraints}

Fuzzy Approach to the transportation problem based on inequality condition mathematically is defined as follows:

Minimize $\tilde{\mathrm{c}}=\sum_{\mathrm{i}=1}^{\mathrm{m}} \sum_{\mathrm{j}=1}^{\mathrm{n}} \tilde{\mathrm{c}}_{\mathrm{ij}} \mathrm{x}_{\mathrm{ij}}$

subject to constraints

$$
\begin{aligned}
& \sum_{j=1}^{n} x_{i j} \leq \tilde{a}_{i} \quad i=1,2, \ldots, m \\
& \sum_{i=1}^{m} x_{i j} \geq \tilde{b}_{j} \quad j=1,2, \ldots, n \\
& x_{i j} \geq 0 \quad i=1,2, \ldots, m ; j=1,2, \ldots, n
\end{aligned}
$$

Where $\tilde{c}_{\mathrm{ij}}=\left(\mathrm{m}_{\mathrm{ij}}, \mathrm{n}_{\mathrm{ij}}, \alpha_{\mathrm{ij}}, \beta_{\mathrm{ij}}\right)_{\mathrm{L}-\mathrm{R}}$ : Fuzzy payment from $\mathrm{i}^{\text {th }}$ source to $\mathrm{j}^{\text {th }}$ destination.

$\mathrm{L}(\mathrm{x})=\mathrm{R}(\mathrm{x})=\max \{0,1-|\mathrm{x}|\} \quad$ or $\quad \mathrm{L}(\mathrm{x})=\mathrm{R}(\mathrm{x})=\mathrm{e}^{-\mathrm{x}}$ or

$\mathrm{L}(\mathrm{x})=\max \{0,1-|\mathrm{x}|\}$ and $\mathrm{R}(\mathrm{x})=\mathrm{e}^{-\mathrm{x}}$ or

$\mathrm{L}(\mathrm{x})=\mathrm{e}^{-\mathrm{x}}$ and $\mathrm{R}(\mathrm{x})=\max \{0,1-|\mathrm{x}|\}$

$\sum_{i=1}^{m} \sum_{j=1}^{n} \tilde{c}_{i j} x_{i j}:$ Total fuzzy cost from $i^{\text {th }}$ source to $j^{\text {th }}$

destination.

where $\tilde{\mathrm{a}}_{\mathrm{i}}=$ fuzzy quantity of supply $\mathrm{i}$,

$\tilde{b}_{j}=$ fuzzy quantity of demand $j$,

$\tilde{\mathrm{c}}_{\mathrm{ij}}=$ the fuzzy cost from $\mathrm{i}^{\text {th }}$ source to $\mathrm{j}^{\text {th }}$ destination.

$\mathrm{L}_{\mathrm{ij}}(\mathrm{x})=$ left shape functions,

$\mathrm{R}_{\mathrm{ij}}(\mathrm{x})=$ right shape functions

All $\tilde{a}_{\mathrm{i}}, \tilde{\mathrm{b}}_{\mathrm{j}}, \tilde{\mathrm{c}}_{\mathrm{ij}}$ denotes a non-negative L-R fuzzy numbers.

\section{PROPOSED METHOD TO FIND THE OPTIMAL SOLUTION FOR FUZZY TRANSPORTATION MODELS}

The proposed method to solve the optimal solution for transportation models using fuzzy linear programming is as follows:

Step 1: Choose a fuzzy transportation problem with equality condition or inequality condition.

Case (i) If the chosen problem is fuzzy transportation problem with equality condition, then consider the model as proposed in section 3.1.

Case (ii) If the chosen problem is fuzzy transportation problem with inequality condition, then consider the model as proposed in section 3.2.

Step2: Based on the problem chosen in step 1 convert it into the following crisp linear programming problem:

Case (a): If the model falls under case (i) of step 1 then convert the fuzzy transportation problem into the following crisp linear programming problem

$$
\text { Minimize } c=\sum_{i=1}^{m} \sum_{j=1}^{n} R\left(\tilde{c}_{i j}\right) x_{i j}
$$

subject to the constraints

$$
\begin{aligned}
& \sum_{j=1}^{n} x_{i j} \cong R\left(\tilde{a}_{i}\right) \quad i=1,2, \ldots, m \\
& \sum_{i=1}^{m} x_{i j} \cong R\left(\tilde{b}_{j}\right) \quad j=1,2, \ldots, n \\
& x_{i j} \geq 0 \quad i=1,2, \ldots, m ; j=1,2, \ldots, n
\end{aligned}
$$

Case (b): If the model falls under case (ii) of step1 then convert the fuzzy transportation problem into the following crisp linear programming problem

$$
\begin{aligned}
& \text { Minimize } c=\sum_{i=1}^{m} \sum_{j=1}^{n} R\left(\tilde{c}_{i j}\right) x_{i j} \\
& \text { subject to the constraints } \\
& \sum_{j=1}^{n} x_{i j} \leq R\left(\tilde{a}_{i}\right) \quad i=1,2, \ldots, m \\
& \sum_{i=1}^{m} x_{i j} \geq R\left(\tilde{b}_{j}\right) \quad j=1,2, \ldots, n \\
& x_{i j} \geq 0 i=1,2, \ldots, m ; j=1,2, \ldots, n
\end{aligned}
$$

Step3: Using Definition 2 and Section 2.2, the values of $\mathrm{R}\left(\tilde{\mathrm{c}}_{\mathrm{ij}}\right), \mathrm{R}\left(\tilde{\mathrm{a}}_{\mathrm{i}}\right)$ and $\mathrm{R}\left(\tilde{\mathrm{b}}_{\mathrm{j}}\right), \forall \mathrm{i}, \mathrm{j}$ are calculated.

Using the values of $R\left(\tilde{c}_{i j}\right), R\left(\tilde{a}_{i}\right)$ and $R\left(\tilde{b}_{j}\right)$, the crisp linear programming problem obtained in step 2 may be written as: Minimize:

$\left(\mathrm{R}\left(\mathrm{c}_{11}\right)\right) \mathrm{x}_{11} \oplus\left(\mathrm{R}\left(\mathrm{c}_{12}\right)\right) \mathrm{x}_{12} \oplus\left(\mathrm{R}\left(\mathrm{c}_{13}\right)\right) \mathrm{x}_{13}$

$\oplus\left(\mathrm{R}\left(\mathrm{c}_{21}\right)\right) \mathrm{x}_{21} \oplus\left(\mathrm{R}\left(\mathrm{c}_{22}\right)\right) \mathrm{x}_{22} \oplus\left(\mathrm{R}\left(\mathrm{c}_{23}\right)\right) \mathrm{x}_{23}$

$\oplus\left(\mathrm{R}\left(\mathrm{c}_{31}\right)\right) \mathrm{x}_{31} \oplus\left(\mathrm{R}\left(\mathrm{c}_{32}\right)\right) \mathrm{x}_{32} \oplus\left(\mathrm{R}\left(\mathrm{c}_{33}\right)\right) \mathrm{x}_{33}$ subject to :

$\mathrm{x}_{11}+\mathrm{x}_{21}+\mathrm{x}_{31}=\mathrm{R}\left(\mathrm{a}_{1}\right), \mathrm{x}_{12}+\mathrm{x}_{22}+\mathrm{x}_{32}=\mathrm{R}\left(\mathrm{a}_{2}\right)$

$\mathrm{x}_{13}+\mathrm{x}_{23}+\mathrm{x}_{33}=\mathrm{R}\left(\mathrm{a}_{3}\right), \mathrm{x}_{11}+\mathrm{x}_{12}+\mathrm{x}_{13}=\mathrm{R}\left(\mathrm{b}_{1}\right)$

$\mathrm{x}_{21}+\mathrm{x}_{22}+\mathrm{x}_{23}=\mathrm{R}\left(\mathrm{b}_{2}\right), \mathrm{x}_{31}+\mathrm{x}_{32}+\mathrm{x}_{33}=\mathrm{R}\left(\mathrm{b}_{3}\right) \mathrm{x}_{\mathrm{ij}} \geq 0$, for all $\mathrm{i}=1,2,3$ and $\mathrm{j}=1,2,3$.

Step 4: Solve crisp linear programming problem obtained in Step 3 to find the optimal solution $\left\{\mathrm{x}_{\mathrm{ij}}\right\}$

Step 5: Solve the minimum fuzzy transportation cost by substituting the optimal solution obtained in step 4 in the objective function of step1.

\section{NUMERICAL EXAMPLE}

To illustrate the proposed models, consider a fuzzy transportation problem with three sources and three destinations. The cost coefficients, the supply and demand in fuzzy transportation problem are considered as L-R fuzzy numbers. The fuzzy transportation problem with fuzzy cost is shown in Table III.

\subsection{Optimal solution using the model based on fuzzy linear programming formulation with equality condition}

The optimal solution of fuzzy transportation problem with equality condition using various cases may be obtained by using the following steps of the proposed method:

Case (i) $\mathrm{L}(\mathrm{x})=\mathrm{R}(\mathrm{x})=\max \{0,1-|\mathrm{x}|\}$

Step 1: The fuzzy linear programming formulation of the fuzzy transportation problem given in Table III 
Minimize

$\left((4,9,3,10)_{\mathrm{L}(1,1)-\mathrm{R}(1,1)}\right) \mathrm{x}_{11} \oplus\left((2,5,1,4)_{\mathrm{L}(1,2)-\mathrm{R}(1,2)}\right) \mathrm{x}_{12} \oplus$ $\left((5,8,3,10)_{\mathrm{L}(1,3)-\mathrm{R}(1,3)}\right) \mathrm{x}_{13} \oplus\left((9,12,1,14)_{\mathrm{L}(2,1)-\mathrm{R}(2,1)}\right) \mathrm{x}_{21}$ $\oplus\left((5,8,2,4)_{\mathrm{L}(2,2)-\mathrm{R}(2,2)}\right) \mathrm{x}_{22} \oplus\left((9,13,2,15)_{\mathrm{L}(2,3)-\mathrm{R}(1,2)}\right) \mathrm{x}_{23} \oplus$ $\left.\left((12,20,1,7)_{\mathrm{L}(3,1)-\mathrm{R}(3,1)}\right) \mathrm{x}_{31} \oplus((5,10,0,5))_{\mathrm{L}(3,2)-\mathrm{R}(3,2)}\right) \mathrm{x}_{32} \oplus$ $\left((5,8,1,3)_{\mathrm{L}(3,3)-\mathrm{R}(3,3)}\right) \mathrm{x}_{33}$

subject to :

$\mathrm{x}_{11}+\mathrm{x}_{21}+\mathrm{x}_{31}=\tilde{\mathrm{a}}_{1}, \mathrm{x}_{12}+\mathrm{x}_{22}+\mathrm{x}_{32}=\tilde{\mathrm{a}}_{2}$

$x_{13}+x_{23}+x_{33}=\tilde{a}_{3}, x_{11}+x_{12}+x_{13}=\tilde{b}_{1}$

$\mathrm{x}_{21}+\mathrm{x}_{22}+\mathrm{x}_{23}=\tilde{\mathrm{b}}_{2}, \mathrm{x}_{31}+\mathrm{x}_{32}+\mathrm{x}_{33}=\tilde{\mathrm{b}}_{3}$

$\mathrm{x}_{\mathrm{ij}} \geq 0$

Step 2: Using Step2 of the proposed method, the formulated fuzzy linear programming problem is converted into the following crisp linear programming problem:

Minimize:

$(\mathrm{R}(4,9,3,10)) \mathrm{x}_{11} \oplus(\mathrm{R}(2,5,1,4)) \mathrm{x}_{12} \oplus$

$(\mathrm{R}(5,8,3,10)) \mathrm{x}_{13} \oplus(\mathrm{R}(9,12,1,14)) \mathrm{x}_{21} \oplus$

$(\mathrm{R}(5,8,2,4)) \mathrm{x}_{22} \oplus(\mathrm{R}(9,13,2,15)) \mathrm{x}_{2} \oplus$

$(\mathrm{R}(12,20,1,7)) \mathrm{x}_{31} \oplus(\mathrm{R}(5,10,0,5)) \mathrm{x}_{32} \oplus$

$(\mathrm{R}(5,8,1,3)) \mathrm{x}_{33}$

subject to :

$\mathrm{x}_{11}+\mathrm{x}_{21}+\mathrm{x}_{31}=\mathrm{R}\left(\tilde{\mathrm{a}}_{1}\right), \mathrm{x}_{12}+\mathrm{x}_{22}+\mathrm{x}_{32}=\mathrm{R}\left(\tilde{\mathrm{a}}_{2}\right)$

$x_{13}+x_{23}+x_{33}=R\left(\tilde{a}_{3}\right), x_{11}+x_{12}+x_{13}=R\left(\tilde{b}_{1}\right)$

$\mathrm{x}_{21}+\mathrm{x}_{22}+\mathrm{x}_{23}=\mathrm{R}\left(\tilde{\mathrm{b}}_{2}\right), \mathrm{x}_{31}+\mathrm{x}_{32}+\mathrm{x}_{33}=\mathrm{R}\left(\tilde{\mathrm{b}}_{3}\right)$

$\mathrm{x}_{\mathrm{ij}} \geq 0$

Step 3: Using Definition 2 and Section 2.2, the values of $\mathrm{R}\left(\tilde{\mathrm{c}}_{\mathrm{ij}}\right), \mathrm{R}\left(\tilde{\mathrm{a}}_{\mathrm{i}}\right)$ and $\mathrm{R}\left(\tilde{\mathrm{b}}_{\mathrm{j}}\right), \forall \mathrm{i}, \mathrm{j}$ are:

$\mathrm{R}\left(\tilde{\mathrm{c}}_{11}\right)=8.25, \mathrm{R}\left(\tilde{\mathrm{c}}_{12}\right)=4.25, \quad \mathrm{R}\left(\tilde{\mathrm{c}}_{13}\right)=8.25, \quad \mathrm{R}\left(\tilde{\mathrm{c}}_{21}\right)=13.75$,

$\mathrm{R}\left(\tilde{\mathrm{c}}_{22}\right)=7, \mathrm{R}\left(\tilde{\mathrm{c}}_{23}\right)=14.25, \mathrm{R}\left(\tilde{\mathrm{c}}_{31}\right)=17.5, \mathrm{R}\left(\tilde{\mathrm{c}}_{32}\right)=8.75$,

$\mathrm{R}\left(\tilde{\mathrm{c}}_{33}\right)=7$ and $\mathrm{R}\left(\tilde{\mathrm{a}}_{1}\right)=4.5, \mathrm{R}\left(\tilde{\mathrm{a}}_{2}\right)=7.25, \mathrm{R}\left(\tilde{\mathrm{a}}_{3}\right)=7$,

$\mathrm{R}\left(\tilde{\mathrm{b}}_{1}\right)=7, \mathrm{R}\left(\tilde{\mathrm{b}}_{2}\right)=6.25, \mathrm{R}\left(\tilde{\mathrm{b}}_{3}\right)=5$

Using the values of $R\left(\tilde{c}_{i j}\right), R\left(\tilde{a}_{i}\right)$ and $R\left(\tilde{b}_{j}\right)$, the crisp linear programming problem obtained in step 2 may be written as: Minimize:

$(8.25) \mathrm{x}_{11} \oplus(4.25) \mathrm{x}_{12} \oplus(8.25) \mathrm{x}_{13} \oplus(13.75) \mathrm{x}_{21} \oplus(7) \mathrm{x}_{22} \oplus$ $(14.25) \mathrm{x}_{23} \oplus(17.5) \mathrm{x}_{31} \oplus(8.75) \mathrm{x}_{32} \oplus(7) \mathrm{x}_{33}$ subject to

$\mathrm{x}_{11}+\mathrm{x}_{21}+\mathrm{x}_{31}=4.5, \mathrm{x}_{12}+\mathrm{x}_{22}+\mathrm{x}_{32}=7.25$

$\mathrm{x}_{13}+\mathrm{x}_{23}+\mathrm{x}_{33}=7, \quad \mathrm{x}_{11}+\mathrm{x}_{12}+\mathrm{x}_{13}=7$

$\mathrm{x}_{21}+\mathrm{x}_{22}+\mathrm{x}_{23}=6.25, \mathrm{x}_{31}+\mathrm{x}_{32}+\mathrm{x}_{33}=5$

$x_{i j} \geq 0$, for all $i=1,2,3$ and $j=1,2,3$.

Step 4: Solving the crisp linear programming problem, obtained in step3, the optimal solution is

$\mathrm{x}_{11}=3.92, \mathrm{x}_{12}=4.25, \mathrm{x}_{13}=8.25, \mathrm{x}_{22}=6.25, \mathrm{x}_{31}=0.08$,

$\mathrm{x}_{33}=4.92$

Step 5: The minimum fuzzy transportation cost by substituting the optimal solution obtained in step 4 in the objective function of step1 is $(84.89,147.88,36.50,104.32)$.

Case (ii) $\mathrm{L}(\mathrm{x})=\mathrm{R}(\mathrm{x})=\mathrm{e}^{-\mathrm{x}}$

Step 1: The fuzzy linear programming formulation of the fuzzy transportation problem given in Table III Minimize:
$\left((4,9,3,10)_{\mathrm{L}(1,1)-\mathrm{R}(1,1)}\right) \mathrm{x}_{11} \oplus\left((2,5,1,4)_{\mathrm{L}(1,2)-\mathrm{R}(1,2)}\right) \mathrm{x}_{12} \oplus$

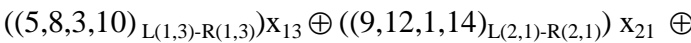

$\left((5,8,2,4)_{\mathrm{L}(2,2)-\mathrm{R}(2,2)}\right) \mathrm{x}_{22} \oplus\left((9,13,2,15)_{\mathrm{L}(2,3)-\mathrm{R}(1,2)}\right) \mathrm{x}_{23}$

$\oplus\left((12,20,1,7)_{\mathrm{L}(3,1)-\mathrm{R}(3,1)}\right) \mathrm{x}_{31} \oplus\left((5,10,0,5)_{\mathrm{L}(3,2)-\mathrm{R}(3,2)}\right) \mathrm{x}_{32} \oplus$

$\left((5,8,1,3)_{\mathrm{L}(3,3)-\mathrm{R}(3,3)}\right) \mathrm{x}_{33}$

subject to :

$\mathrm{x}_{11}+\mathrm{x}_{21}+\mathrm{x}_{31}=\tilde{\mathrm{a}}_{1}, \mathrm{x}_{12}+\mathrm{x}_{22}+\mathrm{x}_{32}=\tilde{\mathrm{a}}_{2}$

$\mathrm{x}_{13}+\mathrm{x}_{23}+\mathrm{x}_{33}=\tilde{\mathrm{a}}_{3}, \mathrm{x}_{11}+\mathrm{x}_{12}+\mathrm{x}_{13}=\tilde{\mathrm{b}}_{1}$

$x_{21}+x_{22}+x_{23}=\tilde{b}_{2}, x_{31}+x_{32}+x_{33}=\tilde{b}_{3}$

$\mathrm{x}_{\mathrm{ij}} \geq 0$

Step 2: Using Step2 of the proposed method, the formulated fuzzy linear programming problem is converted into the following crisp linear programming problem:

Minimize:

$(\mathrm{R}(4,9,3,10)) \mathrm{x}_{11} \oplus(\mathrm{R}(2,5,1,4)) \mathrm{x}_{12} \oplus$

$(\mathrm{R}(5,8,3,10)) \mathrm{x}_{13} \oplus(\mathrm{R}(9,12,1,14)) \mathrm{x}_{21} \oplus$

$(\mathrm{R}(5,8,2,4)) \mathrm{x}_{22} \oplus(\mathrm{R}(9,13,2,15)) \mathrm{x}_{23} \oplus$

$(\mathrm{R}(12,20,1,7)) \mathrm{x}_{31} \oplus(\mathrm{R}(5,10,0,5)) \mathrm{x}_{32} \oplus$

$(\mathrm{R}(5,8,1,3)) \mathrm{x}_{33}$

subject to :

$\mathrm{x}_{11}+\mathrm{x}_{21}+\mathrm{x}_{31}=\mathrm{R}\left(\tilde{\mathrm{a}}_{1}\right), \mathrm{x}_{12}+\mathrm{x}_{22}+\mathrm{x}_{32}=\mathrm{R}\left(\tilde{\mathrm{a}}_{2}\right)$

$\mathrm{x}_{13}+\mathrm{x}_{23}+\mathrm{x}_{33}=\mathrm{R}\left(\tilde{\mathrm{a}}_{3}\right), \mathrm{x}_{11}+\mathrm{x}_{12}+\mathrm{x}_{13}=\mathrm{R}\left(\tilde{\mathrm{b}}_{1}\right)$

$\mathrm{x}_{21}+\mathrm{x}_{22}+\mathrm{x}_{23}=\mathrm{R}\left(\tilde{\mathrm{b}}_{2}\right), \mathrm{x}_{31}+\mathrm{x}_{32}+\mathrm{x}_{33}=\mathrm{R}\left(\tilde{\mathrm{b}}_{3}\right)$

$\mathrm{x}_{\mathrm{ij}} \geq 0$

Step 3: Using Definition 2 and Section 2.2, the values of $\mathrm{R}\left(\tilde{\mathrm{c}}_{\mathrm{ij}}\right), \mathrm{R}\left(\tilde{\mathrm{a}}_{\mathrm{i}}\right)$ and $\mathrm{R}\left(\tilde{\mathrm{b}}_{\mathrm{j}}\right), \forall \mathrm{i}, \mathrm{j}$ are:

$\mathrm{R}\left(\tilde{\mathrm{c}}_{11}\right)=10, \mathrm{R}\left(\tilde{\mathrm{c}}_{12}\right)=5, \mathrm{R}\left(\tilde{\mathrm{c}}_{13}\right)=10, \mathrm{R}\left(\tilde{\mathrm{c}}_{21}\right)=17, \mathrm{R}\left(\tilde{\mathrm{c}}_{22}\right)=7.5$, $\mathrm{R}\left(\tilde{c}_{23}\right)=17.5, \mathrm{R}\left(\tilde{c}_{31}\right)=19, \mathrm{R}\left(\tilde{c}_{32}\right)=10, \mathrm{R}\left(\tilde{c}_{33}\right)=7.5$ and

$\left.\mathrm{R}\left(\tilde{\mathrm{a}}_{1}\right)=5, \mathrm{R}\left(\tilde{\mathrm{a}}_{2}\right)=7 \mathrm{R}\left(\tilde{\mathrm{a}}_{3}\right)=7.5, \mathrm{R}\left(\tilde{\mathrm{b}}_{1}\right)\right)=7.5, \mathrm{R}\left(\tilde{\mathrm{b}}_{2}\right)=7$, $\mathrm{R}\left(\tilde{\mathrm{b}}_{3}\right)=5$

Using the values of $R\left(\tilde{c}_{i j}\right), R\left(\tilde{a}_{i}\right)$ and $R\left(\tilde{b}_{j}\right)$, the crisp linear programming problem obtained in step2 may be written as: Minimize:

$(10) \mathrm{x}_{11} \oplus(5) \mathrm{x}_{12} \oplus(10) \mathrm{x}_{13} \oplus(17) \mathrm{x}_{21} \oplus(7.5) \mathrm{x}_{22} \oplus(17.5) \mathrm{x}_{23}$ $\oplus(19) \mathrm{x}_{31} \oplus(10) \mathrm{x}_{32} \oplus(7.5) \mathrm{x}_{33}$

subject to :

$$
\begin{aligned}
& x_{11}+x_{21}+x_{31}=5, \quad x_{12}+x_{22}+x_{32}=7 \\
& x_{13}+x_{23}+x_{33}=7.5, \quad x_{11}+x_{12}+x_{13}=7.5 \\
& x_{21}+x_{22}+x_{23}=7, \quad x_{31}+x_{32}+x_{33}=5 \\
& x_{i j} \geq 0, \text { for all } i=1,2,3 \text { and } j=1,2,3 .
\end{aligned}
$$

Step 4: Solving the crisp linear programming problem, obtained in step3, the optimal solution is $\mathrm{x}_{11}=5, \mathrm{x}_{13}=2.50, \mathrm{x}_{22}=7.0, \mathrm{x}_{33}=5$

Step 5: The minimum fuzzy transportation cost by substituting the optimal solution obtained in step4 in the objective function of step1 is $(92.5,161,39.5,118)$.

Case (iii) $\mathrm{L}(\mathrm{x})=\max \{0,1-|\mathrm{x}|\}$ and $\mathrm{R}(\mathrm{x})=\mathrm{e}^{-\mathrm{x}}$

Step 1: The fuzzy linear programming formulation of the fuzzy transportation problem given in Table III Minimize:

$\left((4,9,3,10)_{\mathrm{L}(1,1)-\mathrm{R}(1,1)}\right) \mathrm{x}_{11} \oplus\left((2,5,1,4)_{\mathrm{L}(1,2)-\mathrm{R}(1,2)}\right) \mathrm{x}_{12} \oplus$ $\left((5,8,3,10)_{\mathrm{L}(1,3)-\mathrm{R}(1,3)}\right) \mathrm{x}_{13} \oplus\left((9,12,1,14)_{\mathrm{L}(2,1)-\mathrm{R}(2,1)}\right) \mathrm{x}_{21} \oplus$ 
$\left((5,8,2,4)_{\mathrm{L}(2,2)-\mathrm{R}(2,2)}\right) \mathrm{x}_{22} \oplus\left((9,13,2,15)_{\mathrm{L}(2,3)-\mathrm{R}(1,2)}\right) \mathrm{x}_{23} \oplus$ $\left((12,20,1,7)_{\mathrm{L}(3,1)-\mathrm{R}(3,1)}\right) \mathrm{x}_{31} \oplus\left((5,10,0,5)_{\mathrm{L}(3,2)-\mathrm{R}(3,2)}\right) \mathrm{x}_{32} \oplus$ $\left((5,8,1,3)_{\mathrm{L}(3,3)-\mathrm{R}(3,3)}\right) \mathrm{x}_{33}$ subject to :

$\mathrm{x}_{11}+\mathrm{x}_{21}+\mathrm{x}_{31}=\tilde{\mathrm{a}}_{1}, \mathrm{x}_{12}+\mathrm{x}_{22}+\mathrm{x}_{32}=\tilde{\mathrm{a}}_{2}$

$x_{13}+x_{23}+x_{33}=\tilde{a}_{3}, x_{11}+x_{12}+x_{13}=\tilde{b}_{1}$

$\mathrm{x}_{21}+\mathrm{x}_{22}+\mathrm{x}_{23}=\tilde{\mathrm{b}}_{2}, \mathrm{x}_{31}+\mathrm{x}_{32}+\mathrm{x}_{33}=\tilde{\mathrm{b}}_{3}$

$\mathrm{x}_{\mathrm{ij}} \geq 0$

Step 2: Using Step2 of the proposed method, the formulated fuzzy linear programming problem is converted into the following crisp linear programming problem:

\section{Minimize:}

$(\mathrm{R}(4,9,3,10)) \mathrm{x}_{11} \oplus(\mathrm{R}(2,5,1,4)) \mathrm{x}_{12} \oplus$

$(\mathrm{R}(5,8,3,10)) \mathrm{x}_{13} \oplus(\mathrm{R}(9,12,1,14)) \mathrm{x}_{21} \oplus$

$(\mathrm{R}(5,8,2,4)) \mathrm{x}_{22} \oplus(\mathrm{R}(9,13,2,15)) \mathrm{x}_{23} \oplus$

$(\mathrm{R}(12,20,1,7)) \mathrm{x}_{31} \oplus(\mathrm{R}(5,10,0,5)) \mathrm{x}_{32} \oplus$

$(\mathrm{R}(5,8,1,3)) \mathrm{x}_{33}$

subject to :

$$
\begin{aligned}
& x_{11}+x_{21}+x_{31}=R\left(\tilde{a}_{1}\right), x_{12}+x_{22}+x_{32}=R\left(\tilde{a}_{2}\right) \\
& x_{13}+x_{23}+x_{33}=R\left(\tilde{a}_{3}\right), x_{11}+x_{12}+x_{13}=R\left(\tilde{b}_{1}\right) \\
& x_{21}+x_{22}+x_{23}=R\left(\tilde{b}_{2}\right), x_{31}+x_{32}+x_{33}=R\left(\tilde{b}_{3}\right) \\
& x_{i j} \geq 0
\end{aligned}
$$

Step 3: Using Definition 2 and Section 2.2, the values of $\mathrm{R}\left(\tilde{\mathrm{c}}_{\mathrm{ij}}\right), \mathrm{R}\left(\tilde{\mathrm{a}}_{\mathrm{i}}\right)$ and $\mathrm{R}\left(\tilde{\mathrm{b}}_{\mathrm{j}}\right), \forall \mathrm{i}, \mathrm{j}$ are:

$\mathrm{R}\left(\tilde{\mathrm{c}}_{11}\right)=10.75, \mathrm{R}\left(\tilde{\mathrm{c}}_{12}\right)=5.25, \mathrm{R}\left(\tilde{\mathrm{c}}_{13}\right)=10.75, \mathrm{R}\left(\tilde{\mathrm{c}}_{21}\right)=17.25$, $\mathrm{R}\left(\tilde{\mathrm{c}}_{22}\right)=8, \mathrm{R}\left(\tilde{\mathrm{c}}_{23}\right)=18, \mathrm{R}\left(\tilde{\mathrm{c}}_{31}\right)=19.25, \mathrm{R}\left(\tilde{c}_{32}\right)=10$,

$\mathrm{R}\left(\tilde{\mathrm{c}}_{33}\right)=7.75$ and $\mathrm{R}\left(\tilde{\mathrm{a}}_{1}\right)=6, \quad \mathrm{R}\left(\tilde{\mathrm{a}}_{2}\right)=7.75 \quad \mathrm{R}\left(\tilde{\mathrm{a}}_{3}\right)=7.75$, $\mathrm{R}\left(\tilde{\mathrm{b}}_{1}\right)=8, \mathrm{R}\left(\tilde{\mathrm{b}}_{2}\right)=8, \mathrm{R}\left(\tilde{\mathrm{b}}_{3}\right)=5.5$

Using the values of $R\left(\tilde{c}_{i j}\right), R\left(\tilde{a}_{i}\right)$ and $R\left(\tilde{b}_{j}\right)$, the crisp linear programming problem obtained in step 2 may be written as:

Minimize:

$(10.75) \mathrm{x}_{11} \oplus(5.25) \mathrm{x}_{12} \oplus(10.75) \mathrm{x}_{13}$

$\oplus(17.25) \mathrm{x}_{21} \oplus(8) \mathrm{x}_{22} \oplus(18) \mathrm{x}_{23}$

$\oplus(19.25) \mathrm{x}_{31} \oplus(10) \mathrm{x}_{32} \oplus(7.75) \mathrm{x}_{33}$ subject to :

$\mathrm{x}_{11}+\mathrm{x}_{21}+\mathrm{x}_{31}=6, \mathrm{x}_{12}+\mathrm{x}_{22}+\mathrm{x}_{32}=7.75$

$\mathrm{x}_{13}+\mathrm{x}_{23}+\mathrm{x}_{33}=7.75, \mathrm{x}_{11}+\mathrm{x}_{12}+\mathrm{x}_{13}=8$

$\mathrm{x}_{21}+\mathrm{x}_{22}+\mathrm{x}_{23}=8, \mathrm{x}_{31}+\mathrm{x}_{32}+\mathrm{x}_{33}=5.5$

$x_{i j} \geq 0$, for all $i=1,2,3$ and $j=1,2,3$.

Step 4: Solving the crisp linear programming problem, obtained in step3, the optimal solution is

$\mathrm{x}_{11}=5.75, \mathrm{x}_{13}=2.25, \mathrm{x}_{21}=0.25, \mathrm{x}_{22}=7.75, \mathrm{x}_{33}=5.50$

Step 5: The minimum fuzzy transportation cost by substituting the optimal solution obtained in step4 in the objective function of step1 is $(102.75,178.75,45.25,131)$. Case (iv) $\mathrm{L}(\mathrm{x})=\mathrm{e}^{-\mathrm{x}}$ and $\mathrm{R}(\mathrm{x})=\max \{0,1-|\mathrm{x}|\}$

Step 1: The fuzzy linear programming formulation of the fuzzy transportation problem given in Table III Minimize

$\left((4,9,3,10)_{\mathrm{L}(1,1)-\mathrm{R}(1,1)}\right) \mathrm{x}_{11} \oplus\left((2,5,1,4)_{\mathrm{L}(1,2)-\mathrm{R}(1,2)}\right) \mathrm{x}_{12} \oplus$ $\left((5,8,3,10)_{\mathrm{L}(1,3)-\mathrm{R}(1,3)}\right) \mathrm{x}_{13} \oplus\left((9,12,1,14)_{\mathrm{L}(2,1)-\mathrm{R}(2,1)}\right) \mathrm{x}_{21} \oplus$ $\left((5,8,2,4)_{\mathrm{L}(2,2)-\mathrm{R}(2,2)}\right) \mathrm{x}_{22} \oplus\left((9,13,2,15)_{\mathrm{L}(2,3)-\mathrm{R}(1,2)}\right) \mathrm{x}_{23} \oplus$ $\left((12,20,1,7)_{\mathrm{L}(3,1)-\mathrm{R}(3,1)}\right) \mathrm{x}_{31} \oplus\left((5,10,0,5)_{\mathrm{L}(3,2)-\mathrm{R}(3,2)}\right) \mathrm{x}_{32} \oplus$
$((5,8,1,3) \mathrm{L}(3,3)-\mathrm{R}(3,3)) \mathrm{x}_{33}$

subject to :

$$
\begin{aligned}
& x_{11}+x_{21}+x_{31}=\tilde{a}_{1}, x_{12}+x_{22}+x_{32}=\tilde{a}_{2} \\
& x_{13}+x_{23}+x_{33}=\tilde{a}_{3}, x_{11}+x_{12}+x_{13}=\tilde{b}_{1} \\
& x_{21}+x_{22}+x_{23}=\tilde{b}_{2}, x_{31}+x_{32}+x_{33}=\tilde{b}_{3} \\
& x_{i j} \geq 0
\end{aligned}
$$

Step 2: Using Step2 of the proposed method, the formulated fuzzy linear programming problem is converted into the following crisp linear programming problem:

Minimize:

$(\mathrm{R}(4,9,3,10)) \mathrm{x}_{11} \oplus(\mathrm{R}(2,5,1,4)) \mathrm{x}_{12} \oplus$

$(\mathrm{R}(5,8,3,10)) \mathrm{x}_{13} \oplus(\mathrm{R}(9,12,1,14)) \mathrm{x}_{21} \oplus$

$(\mathrm{R}(5,8,2,4)) \mathrm{x}_{22} \oplus(\mathrm{R}(9,13,2,15)) \mathrm{x}_{23} \oplus$

$(\mathrm{R}(12,20,1,7)) \mathrm{x}_{31} \oplus(\mathrm{R}(5,10,0,5)) \mathrm{x}_{32} \oplus$

$(\mathrm{R}(5,8,1,3)) \mathrm{x}_{33}$

subject to :

$x_{11}+x_{21}+x_{31}=R\left(\tilde{a}_{1}\right), x_{12}+x_{22}+x_{32}=R\left(\tilde{a}_{2}\right)$
$x_{13}+x_{23}+x_{33}=R\left(\tilde{a}_{3}\right), x_{11}+x_{12}+x_{13}=R\left(\tilde{b}_{1}\right)$
$x_{21}+x_{22}+x_{23}=R\left(\tilde{b}_{2}\right), x_{31}+x_{32}+x_{33}=R\left(\tilde{b}_{3}\right) x_{i j} \geq 0$

Step 3: Using Definition 2 and Section 2.2, the values of $R\left(\tilde{c}_{i j}\right), R\left(\tilde{a}_{i}\right)$ and $R\left(\tilde{b}_{j}\right), \forall i, j$ are:

$\mathrm{R}\left(\tilde{\mathrm{c}}_{11}\right)=7.5, \mathrm{R}\left(\tilde{\mathrm{c}}_{12}\right)=4, \mathrm{R}\left(\tilde{\mathrm{c}}_{13}\right)=7.5, \mathrm{R}\left(\tilde{\mathrm{c}}_{21}\right)=13.5$,

$\mathrm{R}\left(\tilde{\mathrm{c}}_{22}\right)=6.5, \quad \mathrm{R}\left(\tilde{\mathrm{c}}_{23}\right)=13.75, \quad \mathrm{R}\left(\tilde{\mathrm{c}}_{31}\right)=17.25, \quad \mathrm{R}\left(\tilde{\mathrm{c}}_{32}\right)=8.75$, $\mathrm{R}\left(\tilde{\mathrm{c}}_{33}\right)=6.75$ and $\mathrm{R}\left(\tilde{\mathrm{a}}_{1}\right)=4.5, \mathrm{R}\left(\tilde{\mathrm{a}}_{2}\right)=6.5, \mathrm{R}\left(\tilde{\mathrm{a}}_{3}\right)=6.75$,

$\mathrm{R}\left(\tilde{\mathrm{b}}_{1}\right)=6.5, \mathrm{R}\left(\tilde{\mathrm{b}}_{2}\right)=6.75, \mathrm{R}\left(\tilde{\mathrm{b}}_{3}\right)=4.5$

Using the values of $R\left(\tilde{c}_{i j}\right), R\left(\tilde{a}_{i}\right)$ and $R\left(\tilde{b}_{j}\right)$, the crisp linear programming problem obtained in step 2 may be written as: Minimize:

$(7.5) \mathrm{x}_{11} \oplus(4) \mathrm{x}_{12} \oplus(7.5) \mathrm{x}_{13} \oplus(13.5) \mathrm{x}_{21} \oplus(6.5) \mathrm{x}_{22}$ $\oplus(13.75) \mathrm{x}_{23} \oplus(17.25) \mathrm{x}_{31} \oplus(8.75) \mathrm{x}_{32} \oplus(6.75) \mathrm{x}_{33}$ subject to :

$\mathrm{x}_{11}+\mathrm{x}_{21}+\mathrm{x}_{31}=4.5, \quad \mathrm{x}_{12}+\mathrm{x}_{22}+\mathrm{x}_{32}=6.5$

$x_{13}+x_{23}+x_{33}=6.75, x_{11}+x_{12}+x_{13}=6.5$

$\mathrm{x}_{21}+\mathrm{x}_{22}+\mathrm{x}_{23}=6.75, \mathrm{x}_{31}+\mathrm{x}_{32}+\mathrm{x}_{33}=4.5$

$x_{i j} \geq 0$, for all $i=1,2,3$ and $j=1,2,3$.

Step 4: Solving the crisp linear programming problem, obtained in step3, the optimal solution is

$\mathrm{x}_{11}=4.25, \mathrm{x}_{13}=2.25, \mathrm{x}_{21}=0.25, \mathrm{x}_{22}=6.50, \mathrm{x}_{33}=4.50$

Step 5: The minimum fuzzy transportation cost by substituting the optimal solution obtained in step4 in the objective function of step1 is $(85.5,147.25,37.25,108)$.

5.2. Optimal solution using the method based on fuzzy linear programming formulation with inequality condition The optimal solution of fuzzy transportation problem with in equality condition may be obtained by using the following steps of the proposed method:

Case (i) $L(x)=R(x)=\max \{0,1-|x|\}$

Step 1: The fuzzy linear programming formulation of the fuzzy transportation problem given in Table III

Minimize:

$\left((4,9,3,10)_{\mathrm{L}(1,1)-\mathrm{R}(1,1)}\right) \mathrm{x}_{11} \oplus\left((2,5,1,4)_{\mathrm{L}(1,2)-\mathrm{R}(1,2)}\right) \mathrm{x}_{12} \oplus$

$\left((5,8,3,10)_{\mathrm{L}(1,3)-\mathrm{R}(1,3)}\right) \mathrm{x}_{13} \oplus\left((9,12,1,14)_{\mathrm{L}(2,1)-\mathrm{R}(2,1)}\right) \mathrm{x}_{21} \oplus$

$\left((5,8,2,4)_{\mathrm{L}(2,2)-\mathrm{R}(2,2)}\right) \mathrm{x}_{22} \oplus\left((9,13,2,15)_{\mathrm{L}(2,3)-\mathrm{R}(1,2)}\right) \mathrm{x}_{23} \oplus$

$\left.((12,20,1,7))_{\mathrm{L}(3,1)-\mathrm{R}(3,1)}\right) \mathrm{x}_{31} \oplus\left((5,10,0,5)_{\mathrm{L}(3,2)-\mathrm{R}(3,2)}\right) \mathrm{x}_{32} \oplus$ 
$((5,8,1,3) \mathrm{L}(3,3)-\mathrm{R}(3,3)) \mathrm{x}_{33}$

subject to :

$\mathrm{x}_{11}+\mathrm{x}_{21}+\mathrm{x}_{31}=\tilde{\mathrm{a}}_{1}, \mathrm{x}_{12}+\mathrm{x}_{22}+\mathrm{x}_{32}=\tilde{\mathrm{a}}_{2}$

$\mathrm{x}_{13}+\mathrm{x}_{23}+\mathrm{x}_{33}=\tilde{\mathrm{a}}_{3}, \mathrm{x}_{11}+\mathrm{x}_{12}+\mathrm{x}_{13}=\tilde{\mathrm{b}}_{1}$

$\mathrm{x}_{21}+\mathrm{x}_{22}+\mathrm{x}_{23}=\tilde{\mathrm{b}}_{2}, \mathrm{x}_{31}+\mathrm{x}_{32}+\mathrm{x}_{33}=\tilde{\mathrm{b}}_{3}$

$\mathrm{x}_{\mathrm{ij}} \geq 0$

Step 2: Using Step2 of the proposed method, the formulated fuzzy linear programming problem is converted into the following crisp linear programming problem:

Minimize:

$(\mathrm{R}(4,9,3,10)) \mathrm{x}_{11} \oplus(\mathrm{R}(2,5,1,4)) \mathrm{x}_{12} \oplus$

$(\mathrm{R}(5,8,3,10)) \mathrm{x}_{13} \oplus(\mathrm{R}(9,12,1,14)) \mathrm{x}_{21} \oplus$

$(\mathrm{R}(5,8,2,4)) \mathrm{x}_{22} \oplus(\mathrm{R}(9,13,2,15)) \mathrm{x}_{23} \oplus$

$(\mathrm{R}(12,20,1,7)) \mathrm{x}_{31} \oplus(\mathrm{R}(5,10,0,5)) \mathrm{x}_{32} \oplus$

$(\mathrm{R}(5,8,1,3)) \mathrm{x}_{33}$

subject to :

$\mathrm{x}_{11}+\mathrm{x}_{21}+\mathrm{x}_{31}=\mathrm{R}\left(\tilde{\mathrm{a}}_{1}\right), \mathrm{x}_{12}+\mathrm{x}_{22}+\mathrm{x}_{32}=\mathrm{R}\left(\tilde{\mathrm{a}}_{2}\right)$

$\mathrm{x}_{13}+\mathrm{x}_{23}+\mathrm{x}_{33}=\mathrm{R}\left(\tilde{\mathrm{a}}_{3}\right), \mathrm{x}_{11}+\mathrm{x}_{12}+\mathrm{x}_{13}=\mathrm{R}\left(\tilde{\mathrm{b}}_{1}\right)$

$\mathrm{x}_{21}+\mathrm{x}_{22}+\mathrm{x}_{23}=\mathrm{R}\left(\tilde{\mathrm{b}}_{2}\right), \mathrm{x}_{31}+\mathrm{x}_{32}+\mathrm{x}_{33}=\mathrm{R}\left(\tilde{\mathrm{b}}_{3}\right)$

$\mathrm{x}_{\mathrm{ij}} \geq 0$

Step 3: Using Definition 2 and Section 2.2, the values of $\mathrm{R}\left(\tilde{\mathrm{c}}_{\mathrm{ij}}\right), \mathrm{R}\left(\tilde{\mathrm{a}}_{\mathrm{i}}\right)$ and $\mathrm{R}\left(\tilde{\mathrm{b}}_{\mathrm{j}}\right), \forall \mathrm{i}, \mathrm{j}$ are: are

$\mathrm{R}\left(\tilde{\mathrm{c}}_{11}\right)=8.25, \quad \mathrm{R}\left(\tilde{\mathrm{c}}_{12}\right)=4.25 \quad \mathrm{R}\left(\tilde{\mathrm{c}}_{13}\right)=8.25, \quad \mathrm{R}\left(\tilde{\mathrm{c}}_{21}\right)=13.75$, $\mathrm{R}\left(\tilde{\mathrm{c}}_{22}\right)=7, \mathrm{R}\left(\tilde{\mathrm{c}}_{23}\right)=14.25, \mathrm{R}\left(\tilde{\mathrm{c}}_{31}\right)=17.5, \mathrm{R}\left(\tilde{\mathrm{c}}_{32}\right)=8.75, \mathrm{R}\left(\tilde{\mathrm{c}}_{33}\right)$ $=7$ and $\quad \mathrm{R}\left(\tilde{\mathrm{a}}_{1}\right)=4.5, \quad \mathrm{R}\left(\tilde{\mathrm{a}}_{2}\right)=7.25, \quad \mathrm{R}\left(\tilde{\mathrm{a}}_{3}\right)=7, \mathrm{R}\left(\tilde{\mathrm{b}}_{1}\right)=7$, $\mathrm{R}\left(\tilde{\mathrm{b}}_{2}\right)=6.25, \mathrm{R}\left(\tilde{\mathrm{b}}_{3}\right)=5$

Using the values of $R\left(\tilde{c}_{i j}\right), R\left(\tilde{a}_{i}\right)$ and $R\left(\tilde{b}_{j}\right)$, the crisp linear programming problem obtained in step 2 may be written as: Minimize:

$(8.25) \mathrm{x}_{11} \oplus(4.25) \mathrm{x}_{12} \oplus(8.25) \mathrm{x}_{13} \oplus(13.75) \mathrm{x}_{21} \oplus(7) \mathrm{x}_{22} \oplus$ $(14.25) \mathrm{x}_{23} \oplus(17.5) \mathrm{x}_{31} \oplus(8.75) \mathrm{x}_{32} \oplus(7) \mathrm{x}_{33}$ subject to :

$\mathrm{x}_{11}+\mathrm{x}_{21}+\mathrm{x}_{31}=4.5, \quad \mathrm{x}_{12}+\mathrm{x}_{22}+\mathrm{x}_{32}=7.25$

$\mathrm{x}_{13}+\mathrm{x}_{23}+\mathrm{x}_{33}=7, \quad \mathrm{x}_{11}+\mathrm{x}_{12}+\mathrm{x}_{13}=7$

$\mathrm{x}_{21}+\mathrm{x}_{22}+\mathrm{x}_{23}=6.25, \mathrm{x}_{31}+\mathrm{x}_{32}+\mathrm{x}_{33}=5$

$\mathrm{x}_{\mathrm{ij}} \geq 0$, for all $\mathrm{i}=1,2,3$ and $\mathrm{j}=1,2,3$.

Step 4: Solving the crisp linear programming problem, obtained in step3, the optimal solution is

$\mathrm{x}_{11}=4, \mathrm{x}_{12}=1, \mathrm{x}_{13}=2, \mathrm{x}_{22}=6.25, \mathrm{x}_{33}=5$

Step 5: The minimum fuzzy transportation cost by substituting the optimal solution obtained in step4 in the objective function of step1 is $(90.75,163.25,39.75,117)$.

Case (ii) $\mathrm{L}(\mathrm{x})=\mathrm{R}(\mathrm{x})=\mathrm{e}^{-\mathrm{x}}$

Step 1: The fuzzy linear programming formulation of the fuzzy transportation problem given in Table III

Minimize:

$\left((4,9,3,10)_{\mathrm{L}(1,1)-\mathrm{R}(1,1)}\right) \mathrm{x}_{11} \oplus\left((2,5,1,4)_{\mathrm{L}(1,2)-\mathrm{R}(1,2)}\right) \mathrm{x}_{12} \oplus$

$\left((5,8,3,10)_{\mathrm{L}(1,3)-\mathrm{R}(1,3)}\right) \mathrm{x}_{13} \oplus\left((9,12,1,14)_{\mathrm{L}(2,1)-\mathrm{R}(2,1)}\right) \mathrm{x}_{21} \oplus$

$\left((5,8,2,4)_{\mathrm{L}(2,2)-\mathrm{R}(2,2)}\right) \mathrm{x}_{22} \oplus\left((9,13,2,15)_{\mathrm{L}(2,3)-\mathrm{R}(1,2)}\right) \mathrm{x}_{23} \oplus$

$\left((12,20,1,7)_{\mathrm{L}(3,1)-\mathrm{R}(3,1)}\right) \mathrm{x}_{31} \oplus\left((5,10,0,5)_{\mathrm{L}(3,2)-\mathrm{R}(3,2)}\right) \mathrm{x}_{32} \oplus$

$((5,8,1,3) \mathrm{L}(3,3)-\mathrm{R}(3,3)) \mathrm{x}_{33}$

subject to :

$\mathrm{x}_{11}+\mathrm{x}_{21}+\mathrm{x}_{31}=\tilde{\mathrm{a}}_{1}, \mathrm{x}_{12}+\mathrm{x}_{22}+\mathrm{x}_{32}=\tilde{\mathrm{a}}_{2}$ $x_{13}+x_{23}+x_{33}=\tilde{a}_{3}, x_{11}+x_{12}+x_{13}=\tilde{b}_{1}$
$x_{21}+x_{22}+x_{23}=\tilde{b}_{2}, x_{31}+x_{32}+x_{33}=\tilde{b}_{3}$
$x_{i j} \geq 0$

Step 2: Using Step2 of the proposed method, the formulated fuzzy linear programming problem is converted into the following crisp linear programming problem:

Minimize:

$(\mathrm{R}(4,9,3,10)) \mathrm{x}_{11} \oplus(\mathrm{R}(2,5,1,4)) \mathrm{x}_{12} \oplus$

$(\mathrm{R}(5,8,3,10)) \mathrm{x}_{13} \oplus(\mathrm{R}(9,12,1,14)) \mathrm{x}_{21} \oplus$

$(\mathrm{R}(5,8,2,4)) \mathrm{x}_{22} \oplus(\mathrm{R}(9,13,2,15)) \mathrm{x}_{23} \oplus$

$(\mathrm{R}(12,20,1,7)) \mathrm{x}_{31} \oplus(\mathrm{R}(5,10,0,5)) \mathrm{x}_{32} \oplus$

$(\mathrm{R}(5,8,1,3)) \mathrm{x}_{33}$

subject to :

$$
\begin{aligned}
& x_{11}+x_{21}+x_{31}=R\left(\tilde{a}_{1}\right), x_{12}+x_{22}+x_{32}=R\left(\tilde{a}_{2}\right) \\
& x_{13}+x_{23}+x_{33}=R\left(\tilde{a}_{3}\right), x_{11}+x_{12}+x_{13}=R\left(\tilde{b}_{1}\right) \\
& x_{21}+x_{22}+x_{23}=R\left(\tilde{b}_{2}\right), x_{31}+x_{32}+x_{33}=R\left(\tilde{b}_{3}\right) x_{i j} \geq 0
\end{aligned}
$$

Step 3: Using Definition 2 and Section 2.2, the values of $\mathrm{R}\left(\tilde{\mathrm{c}}_{\mathrm{ij}}\right), \mathrm{R}\left(\tilde{\mathrm{a}}_{\mathrm{i}}\right)$ and $\mathrm{R}\left(\tilde{\mathrm{b}}_{\mathrm{j}}\right), \forall \mathrm{i}, \mathrm{j}$ are:

$\mathrm{R}\left(\tilde{\mathrm{c}}_{11}\right)=10, \mathrm{R}\left(\tilde{\mathrm{c}}_{12}\right)=5, \mathrm{R}\left(\tilde{\mathrm{c}}_{13}\right)=10, \mathrm{R}\left(\tilde{\mathrm{c}}_{21}\right)=17, \mathrm{R}\left(\tilde{\mathrm{c}}_{22}\right)=7.5$, $\mathrm{R}\left(\tilde{\mathrm{c}}_{23}\right)=17.5, \mathrm{R}\left(\tilde{\mathrm{c}}_{31}\right)=19, \mathrm{R}\left(\tilde{\mathrm{c}}_{32}\right)=10, \mathrm{R}\left(\tilde{\mathrm{c}}_{33}\right)=7.5$ and $\mathrm{R}\left(\tilde{\mathrm{a}}_{1}\right)=5, \quad \mathrm{R}\left(\tilde{\mathrm{a}}_{2}\right)=7, \quad \mathrm{R}\left(\tilde{\mathrm{a}}_{3}\right)=7.5, \mathrm{R}\left(\tilde{\mathrm{b}}_{1}\right)=7.5, \quad \mathrm{R}\left(\tilde{\mathrm{b}}_{2}\right)=7$, $\mathrm{R}\left(\tilde{\mathrm{b}}_{3}\right)=5$

Using the values of $R\left(\tilde{c}_{i j}\right), R\left(\tilde{a}_{i}\right)$ and $R\left(\tilde{b}_{j}\right)$, the crisp linear programming problem obtained in step 2 may be written as: Minimize:

$(10) \mathrm{x}_{11} \oplus(5) \mathrm{x}_{12} \oplus(10) \mathrm{x}_{13} \oplus(17) \mathrm{x}_{21} \oplus(7.5) \mathrm{x}_{22} \oplus$

$(17.5) \mathrm{x}_{23} \oplus(19) \mathrm{x}_{31} \oplus(10) \mathrm{x}_{32} \oplus(7.5) \mathrm{x}_{33}$ subject to :

$\mathrm{x}_{11}+\mathrm{x}_{21}+\mathrm{x}_{31}=5, \quad \mathrm{x}_{12}+\mathrm{x}_{22}+\mathrm{x}_{32}=7$

$x_{13}+x_{23}+x_{33}=7.5, x_{11}+x_{12}+x_{13}=7.5$

$\mathrm{x}_{21}+\mathrm{x}_{22}+\mathrm{x}_{23}=7, \quad \mathrm{x}_{31}+\mathrm{x}_{32}+\mathrm{x}_{33}=5$

$\mathrm{x}_{\mathrm{ij}} \geq 0$, for all $\mathrm{i}=1,2,3$ and $\mathrm{j}=1,2,3$.

Step 4: Solving the crisp linear programming problem, obtained in step3, the optimal solution is $\mathrm{x}_{11}=5, \mathrm{x}_{13}=2.50, \mathrm{x}_{22}=7.0, \mathrm{x}_{33}=5$

Step 5: The minimum fuzzy transportation cost by substituting the optimal solution obtained in step4 in the objective function of step1 is $(92.5,161,39.5,118)$.

Case (iii) $\mathrm{L}(\mathrm{x})=\max \{0,1-|\mathrm{x}|\}$ and $\mathrm{R}(\mathrm{x})=\mathrm{e}^{-\mathrm{x}}$

Step1: The fuzzy linear programming formulation of the fuzzy transportation problem given in Table III Minimize:

$\left((4,9,3,10)_{\mathrm{L}(1,1)-\mathrm{R}(1,1)}\right) \mathrm{x}_{11} \oplus\left((2,5,1,4)_{\mathrm{L}(1,2)-\mathrm{R}(1,2)}\right) \mathrm{x}_{12} \oplus$

$\left((5,8,3,10)_{\mathrm{L}(1,3)-\mathrm{R}(1,3)}\right) \mathrm{x}_{13} \oplus\left((9,12,1,14)_{\mathrm{L}(2,1)-\mathrm{R}(2,1)}\right) \mathrm{x}_{21} \oplus$ $\left((5,8,2,4)_{\mathrm{L}(2,2)-\mathrm{R}(2,2)}\right) \mathrm{x}_{22} \oplus((9,13,2,15) \mathrm{L}(2,3)-\mathrm{R}(1,2)) \mathrm{x}_{23} \oplus$ $\left((12,20,1,7)_{\mathrm{L}(3,1)-\mathrm{R}(3,1)}\right) \mathrm{x}_{31} \oplus\left((5,10,0,5)_{\mathrm{L}(3,2)-\mathrm{R}(3,2)}\right) \mathrm{x}_{32} \oplus$ $\left((5,8,1,3){ }_{\mathrm{L}(3,3)-\mathrm{R}(3,3)}\right) \mathrm{x}_{33}$ subject to :

$\mathrm{x}_{11}+\mathrm{x}_{21}+\mathrm{x}_{31}=\tilde{\mathrm{a}}_{1}, \mathrm{x}_{12}+\mathrm{x}_{22}+\mathrm{x}_{32}=\tilde{\mathrm{a}}_{2}$

$\mathrm{x}_{13}+\mathrm{x}_{23}+\mathrm{x}_{33}=\tilde{\mathrm{a}}_{3}, \mathrm{x}_{11}+\mathrm{x}_{12}+\mathrm{x}_{13}=\tilde{\mathrm{b}}_{1}$

$\mathrm{x}_{21}+\mathrm{x}_{22}+\mathrm{x}_{23}=\tilde{\mathrm{b}}_{2}, \mathrm{x}_{31}+\mathrm{x}_{32}+\mathrm{x}_{33}=\tilde{\mathrm{b}}_{3}$

$\mathrm{x}_{\mathrm{ij}} \geq 0$ 
Step 2: Using Step2 of the proposed method, the formulated fuzzy linear programming problem is converted into the following crisp linear programming problem:

Minimize:

$(\mathrm{R}(4,9,3,10)) \mathrm{x}_{11} \oplus(\mathrm{R}(2,5,1,4)) \mathrm{x}_{12} \oplus$

$(\mathrm{R}(5,8,3,10)) \mathrm{x}_{13} \oplus(\mathrm{R}(9,12,1,14)) \mathrm{x}_{21} \oplus$

$(\mathrm{R}(5,8,2,4)) \mathrm{x}_{22} \oplus(\mathrm{R}(9,13,2,15)) \mathrm{x}_{23} \oplus$

$(\mathrm{R}(12,20,1,7)) \mathrm{x}_{31} \oplus(\mathrm{R}(5,10,0,5)) \mathrm{x}_{32} \oplus$

$(\mathrm{R}(5,8,1,3)) \mathrm{x}_{33}$

subject to :

$\mathrm{x}_{11}+\mathrm{x}_{21}+\mathrm{x}_{31}=\mathrm{R}\left(\tilde{\mathrm{a}}_{1}\right), \mathrm{x}_{12}+\mathrm{x}_{22}+\mathrm{x}_{32}=\mathrm{R}\left(\tilde{\mathrm{a}}_{2}\right)$

$\mathrm{x}_{13}+\mathrm{x}_{23}+\mathrm{x}_{33}=\mathrm{R}\left(\tilde{\mathrm{a}}_{3}\right), \mathrm{x}_{11}+\mathrm{x}_{12}+\mathrm{x}_{13}=\mathrm{R}\left(\tilde{\mathrm{b}}_{1}\right)$

$\mathrm{x}_{21}+\mathrm{x}_{22}+\mathrm{x}_{23}=\mathrm{R}\left(\tilde{\mathrm{b}}_{2}\right), \mathrm{x}_{31}+\mathrm{x}_{32}+\mathrm{x}_{33}=\mathrm{R}\left(\tilde{\mathrm{b}}_{3}\right)$

$\mathrm{x}_{\mathrm{ij}} \geq 0$

Step 3: Using Definition 2 and Section 2.2, the values of $\mathrm{R}\left(\tilde{\mathrm{c}}_{\mathrm{ij}}\right), \mathrm{R}\left(\tilde{\mathrm{a}}_{\mathrm{i}}\right)$ and $\mathrm{R}\left(\tilde{\mathrm{b}}_{\mathrm{j}}\right), \forall \mathrm{i}, \mathrm{j}$ are:

$\mathrm{R}\left(\tilde{\mathrm{c}}_{11}\right)=10.75, \quad \mathrm{R}\left(\tilde{\mathrm{c}}_{12}\right)=5.25, \mathrm{R}\left(\tilde{\mathrm{c}}_{13}\right)=10.75, \quad \mathrm{R}\left(\tilde{\mathrm{c}}_{21}\right)=17.25$,

$\mathrm{R}\left(\tilde{\mathrm{c}}_{22}\right)=8, \mathrm{R}\left(\tilde{\mathrm{c}}_{23}\right)=18, \mathrm{R}\left(\tilde{\mathrm{c}}_{31}\right)=19.25, \mathrm{R}\left(\tilde{\mathrm{c}}_{32}\right)=10$,

$\mathrm{R}\left(\tilde{c}_{33}\right)=7.75$ and $\mathrm{R}\left(\tilde{\mathrm{a}}_{1}\right)=6, \mathrm{R}\left(\tilde{\mathrm{a}}_{2}\right)=7.75, \mathrm{R}\left(\tilde{\mathrm{a}}_{3}\right)=7.75$,

$\mathrm{R}\left(\tilde{\mathrm{b}}_{1}\right)=8, \mathrm{R}\left(\tilde{\mathrm{b}}_{2}\right)=8, \mathrm{R}\left(\tilde{\mathrm{b}}_{3}\right)=5.5$

Using the values of $R\left(\tilde{c}_{i j}\right), R\left(\tilde{a}_{i}\right)$ and $R\left(\tilde{b}_{j}\right)$, the crisp linear programming problem obtained in step 2 may be written as: Minimize:

$(10.75) \mathrm{x}_{11} \oplus(5.25) \mathrm{x}_{12} \oplus(10.75) \mathrm{x}_{13} \oplus(17.25) \mathrm{x}_{21} \oplus(8) \mathrm{x}_{22}$ $\oplus(18) \mathrm{x}_{23} \oplus(19.25) \mathrm{x}_{31} \oplus(10) \mathrm{x}_{32} \oplus(7.75) \mathrm{x}_{33}$

subject to :

$\mathrm{x}_{11}+\mathrm{x}_{21}+\mathrm{x}_{31}=6, \mathrm{x}_{12}+\mathrm{x}_{22}+\mathrm{x}_{32}=7.75$

$\mathrm{x}_{13}+\mathrm{x}_{23}+\mathrm{x}_{33}=7.75, \mathrm{x}_{11}+\mathrm{x}_{12}+\mathrm{x}_{13}=8$

$\mathrm{x}_{21}+\mathrm{x}_{22}+\mathrm{x}_{23}=8, \mathrm{x}_{31}+\mathrm{x}_{32}+\mathrm{x}_{33}=5.5$

$x_{i j} \geq 0$, for all $i=1,2,3$ and $j=1,2,3$.

Step 4: Solving the crisp linear programming problem, obtained in step3, the optimal solution is

$\mathrm{x}_{11}=5.75, \mathrm{x}_{13}=2.25, \mathrm{x}_{21}=0.25, \mathrm{x}_{22}=7.75, \mathrm{x}_{33}=5.50$

Step 5: The minimum fuzzy transportation cost by substituting the optimal solution obtained in step4 in the objective function of step 1 is $(102.75,178.75,45.25,131)$

Case (iv) $\mathrm{L}(\mathrm{x})=\mathrm{e}^{-\mathrm{x}}$ and $\mathrm{R}(\mathrm{x})=\max \{0,1-|\mathrm{x}|\}$

Step 1: The fuzzy linear programming formulation of the fuzzy transportation problem given in Table III

Minimize:

$\left((4,9,3,10)_{\mathrm{L}(1,1)-\mathrm{R}(1,1)}\right) \mathrm{x}_{11} \oplus\left((2,5,1,4)_{\mathrm{L}(1,2)-\mathrm{R}(1,2)}\right) \mathrm{x}_{12} \oplus$

$\left((5,8,3,10)_{\mathrm{L}(1,3)-\mathrm{R}(1,3)}\right) \mathrm{x}_{13} \oplus\left((9,12,1,14)_{\mathrm{L}(2,1)-\mathrm{R}(2,1)}\right) \mathrm{x}_{21} \oplus$

$\left((5,8,2,4)_{\mathrm{L}(2,2)-\mathrm{R}(2,2)}\right) \mathrm{x}_{22} \oplus\left((9,13,2,15)_{\mathrm{L}(2,3)-\mathrm{R}(1,2)}\right) \mathrm{x}_{23} \oplus$

$\left((12,20,1,7)_{\mathrm{L}(3,1)-\mathrm{R}(3,1)}\right) \mathrm{x}_{31} \oplus((5,10,0,5) \mathrm{L}(3,2)-\mathrm{R}(3,2)) \mathrm{x}_{32} \oplus$

$((5,8,1,3) \mathrm{L}(3,3)-\mathrm{R}(3,3)) \mathrm{x}_{33}$

subject to :

$\mathrm{x}_{11}+\mathrm{x}_{21}+\mathrm{x}_{31}=\tilde{\mathrm{a}}_{1}, \mathrm{x}_{12}+\mathrm{x}_{22}+\mathrm{x}_{32}=\tilde{\mathrm{a}}_{2}$

$x_{13}+x_{23}+x_{33}=\tilde{a}_{3}, x_{11}+x_{12}+x_{13}=\tilde{b}_{1}$

$x_{21}+x_{22}+x_{23}=\tilde{b}_{2}, x_{31}+x_{32}+x_{33}=\tilde{b}_{3}$

$\mathrm{x}_{\mathrm{ij}} \geq 0$

Step 2: Using Step 2 of the proposed method, the formulated fuzzy linear programming problem is converted into the following crisp linear programming problem:
Minimize:

$(\mathrm{R}(4,9,3,10)) \mathrm{x}_{11} \oplus(\mathrm{R}(2,5,1,4)) \mathrm{x}_{12} \oplus$

$(\mathrm{R}(5,8,3,10)) \mathrm{x}_{13} \oplus(\mathrm{R}(9,12,1,14)) \mathrm{x}_{21} \oplus$

$(\mathrm{R}(5,8,2,4)) \mathrm{x}_{22} \oplus(\mathrm{R}(9,13,2,15)) \mathrm{x}_{23} \oplus$

$(\mathrm{R}(12,20,1,7)) \mathrm{x}_{31} \oplus(\mathrm{R}(5,10,0,5)) \mathrm{x}_{32} \oplus$

$(\mathrm{R}(5,8,1,3)) \mathrm{x}_{33}$

subject to :

$$
\begin{aligned}
& \mathrm{x}_{11}+\mathrm{x}_{21}+\mathrm{x}_{31}=\mathrm{R}\left(\tilde{\mathrm{a}}_{1}\right), \mathrm{x}_{12}+\mathrm{x}_{22}+\mathrm{x}_{32}=\mathrm{R}\left(\tilde{\mathrm{a}}_{2}\right) \\
& \mathrm{x}_{13}+\mathrm{x}_{23}+\mathrm{x}_{33}=\mathrm{R}\left(\tilde{\mathrm{a}}_{3}\right), \mathrm{x}_{11}+\mathrm{x}_{12}+\mathrm{x}_{13}=\mathrm{R}\left(\tilde{\mathrm{b}}_{1}\right) \\
& \mathrm{x}_{21}+\mathrm{x}_{22}+\mathrm{x}_{23}=\mathrm{R}\left(\tilde{\mathrm{b}}_{2}\right), \mathrm{x}_{31}+\mathrm{x}_{32}+\mathrm{x}_{33}=\mathrm{R}\left(\tilde{\mathrm{b}}_{3}\right) \\
& \mathrm{x}_{\mathrm{ij}} \geq 0
\end{aligned}
$$

Step 3: Using Definition 2 and Section 2.2, the values of $R\left(\tilde{c}_{i j}\right), R\left(\tilde{a}_{i}\right)$ and $R\left(\tilde{b}_{j}\right), \forall i, j$ are:

$\mathrm{R}\left(\tilde{\mathrm{c}}_{11}\right)=7.5, \mathrm{R}\left(\tilde{\mathrm{c}}_{12}\right)=4, \mathrm{R}\left(\tilde{\mathrm{c}}_{13}\right)=7.5, \mathrm{R}\left(\tilde{\mathrm{c}}_{21}\right)=13.5$,

$\mathrm{R}\left(\tilde{\mathrm{c}}_{22}\right)=6.5, \quad \mathrm{R}\left(\tilde{\mathrm{c}}_{23}\right)=13.75, \quad \mathrm{R}\left(\tilde{\mathrm{c}}_{31}\right)=17.25, \quad \mathrm{R}\left(\tilde{\mathrm{c}}_{32}\right)=8.75$

$\mathrm{R}\left(\tilde{\mathrm{c}}_{33}\right)=6.75$ and $\mathrm{R}\left(\tilde{\mathrm{a}}_{1}\right)=4.5, \mathrm{R}\left(\tilde{\mathrm{a}}_{2}\right)=6.5, \mathrm{R}\left(\tilde{\mathrm{a}}_{3}\right)=6.75$,

$\mathrm{R}\left(\tilde{\mathrm{b}}_{1}\right)=6.5, \mathrm{R}\left(\tilde{\mathrm{b}}_{2}\right)=6.75, \mathrm{R}\left(\tilde{\mathrm{b}}_{3}\right)=4.5$

Using the values of $R\left(\tilde{c}_{i j}\right), R\left(\tilde{a}_{i}\right)$ and $R\left(\tilde{b}_{j}\right)$, the crisp linear programming problem obtained in step 2 may be written as: Minimize:

$(7.5) \mathrm{x}_{11} \oplus(4) \mathrm{x}_{12} \oplus(7.5) \mathrm{x}_{13} \oplus(13.5) \mathrm{x}_{21} \oplus(6.5) \mathrm{x}_{22}$

$\oplus(13.75) \mathrm{x}_{23} \oplus(17.25) \mathrm{x}_{31} \oplus(8.75) \mathrm{x}_{32} \oplus(6.75) \mathrm{x}_{33}$ subject to :

$\mathrm{x}_{11}+\mathrm{x}_{21}+\mathrm{x}_{31}=4.5, \mathrm{x}_{12}+\mathrm{x}_{22}+\mathrm{x}_{32}=6.5$

$\mathrm{x}_{13}+\mathrm{x}_{23}+\mathrm{x}_{33}=6.75, \mathrm{x}_{11}+\mathrm{x}_{12}+\mathrm{x}_{13}=6.5$

$\mathrm{x}_{21}+\mathrm{x}_{22}+\mathrm{x}_{23}=6.75, \mathrm{x}_{31}+\mathrm{x}_{32}+\mathrm{x}_{33}=4.5 \mathrm{x}_{\mathrm{ij}} \geq 0$, for all $\mathrm{i}=1,2,3$ and $\mathrm{j}=1,2,3$.

Step 4: Solving the crisp linear programming problem, obtained in step3, the optimal solution is

$\mathrm{x}_{11}=4.25, \mathrm{x}_{13}=2.25, \mathrm{x}_{21}=0.25, \mathrm{x}_{22}=6.50, \mathrm{x}_{33}=4.50$

Step 5: The minimum fuzzy transportation cost by substituting the optimal solution obtained in step4 in the objective function of step1 is $(85.5,147.25,37.25,108)$.

\section{CONCLUSION}

In this paper two new fuzzy transportation linear programming models are developed: one with equality constraints and other with inequality constraints using L-R fuzzy numbers. The membership function of L-R fuzzy numbers of fuzzy cost are consider to be linear and exponential. A procedure have been developed to derive the fuzzy objective value of the fuzzy transportation problem, in that the cost coefficients and the supply and demand are L-R fuzzy numbers. The two models are illustrated with an example and we observed that the optimal fuzzy transportation cost for the two models slightly varies when linear membership functions are equal and the optimal fuzzy transportation cost is same in case of different membership functions i.e., either linear or exponential membership functions defined on L-R fuzzy numbers. Most of the fuzzy transportation problems reviewed in literature have the negative optimal fuzzy transportation cost but in our proposed method we obtain positive optimal fuzzy transportation cost in all most all cases. 
Table I. Reference functions and their inverses

\begin{tabular}{|c|c|c|}
\hline Function Name & Reference Function (RF) & $\begin{array}{c}\text { Inverse of Reference function } \\
\alpha \in(0,1]\end{array}$ \\
\hline Linear & $\mathrm{RF}_{\mathrm{p}}(\mathrm{x})=\max \{0,1-|\mathrm{x}|\}$ & $\mathrm{RF}_{\mathrm{p}}^{-1}(\mathrm{x})=(1-\alpha)$ \\
\hline Exponential & $\mathrm{RF}_{\mathrm{p}}(\mathrm{x})=\mathrm{e}^{-\mathrm{px}}, \mathrm{p} \geq 1$ & $\mathrm{RF}_{\mathrm{p}}^{-1}(\mathrm{x})=-(\operatorname{In} \alpha) / \mathrm{p}$ \\
\hline Power & $\mathrm{RF}_{\mathrm{p}}(\mathrm{x})=\max \left(0,1-\mathrm{x}^{\mathrm{p}}\right), \mathrm{p} \geq 1$ & $\mathrm{RF}_{\mathrm{p}}^{-1}(\mathrm{x})=\sqrt[\mathrm{p}]{1-\alpha}$ \\
\hline Exponential power & $\mathrm{RF}_{\mathrm{p}}(\mathrm{x})=\mathrm{e}^{-\mathrm{x}^{\mathrm{p}}}, \mathrm{p} \geq 1$ & $\mathrm{RF}_{\mathrm{p}}^{-1}(\mathrm{x})=\sqrt[\mathrm{p}]{-\operatorname{In} \alpha}$ \\
\hline Rational & $\mathrm{RF}_{\mathrm{p}}(\mathrm{x})=1 /\left(1+\mathrm{x}^{\mathrm{p}}\right), \mathrm{p} \geq 1$ & $\mathrm{RF}_{\mathrm{p}}^{-1}(\mathrm{x})=\sqrt[p]{(1-\alpha) / \alpha}$ \\
\hline
\end{tabular}

Table II: Fuzzy transportation model with fuzzy cost

\begin{tabular}{|c|c|c|c|c|c|c|c|}
\hline $\begin{array}{c}\text { Source/ } \\
\text { Destination }\end{array}$ & $\mathbf{1}$ & $\mathbf{2}$ & $\cdots$ & $\mathbf{j}$ & $\cdots$ & $\mathbf{n}$ & Supply \\
\hline $\mathbf{A}$ & $\tilde{\mathrm{c}}_{11}$ & $\tilde{\mathrm{c}}_{12}$ & $\cdots$ & $\tilde{\mathrm{c}}_{1 \mathrm{j}}$ & $\cdots$ & $\tilde{\mathrm{c}}_{1 \mathrm{n}}$ & $\tilde{\mathrm{a}}_{1}$ \\
\hline $\mathbf{B}$ & $\tilde{\mathrm{c}}_{21}$ & $\tilde{\mathrm{c}}_{22}$ & $\cdots$ & $\tilde{\mathrm{c}}_{2 \mathrm{j}}$ & $\cdots$ & $\tilde{\mathrm{c}}_{2 \mathrm{n}}$ & $\tilde{\mathrm{a}}_{2}$ \\
\hline$\ldots$ & $\ldots$ & $\ldots$ & $\cdots$ & $\ldots$ & $\cdots$ & $\ldots$ & $\ldots$ \\
\hline $\mathbf{i}$ & $\tilde{\mathrm{c}}_{\mathrm{i} 1}$ & $\tilde{\mathrm{c}}_{\mathrm{i} 2}$ & $\cdots$ & $\tilde{\mathrm{c}}_{\mathrm{ij}}$ & $\cdots$ & $\tilde{\mathrm{c}}_{\mathrm{in}}$ & $\tilde{\mathrm{a}}_{\mathrm{i}}$ \\
\hline$\ldots$ & $\ldots$ & $\ldots$ & $\cdots$ & $\ldots$ & $\cdots$ & $\ldots$ & $\ldots$ \\
\hline $\mathbf{m}$ & $\tilde{\mathrm{c}}_{\mathrm{m} 1}$ & $\tilde{\mathrm{c}}_{\mathrm{m} 2}$ & $\cdots$ & $\tilde{\mathrm{c}}_{\mathrm{mj}}$ & $\cdots$ & $\tilde{\mathrm{c}}_{\mathrm{mn}}$ & $\tilde{\mathrm{a}}_{\mathrm{m}}$ \\
\hline Demand & $\tilde{\mathrm{b}}_{1}$ & $\tilde{\mathrm{b}}_{2}$ & $\cdots$ & $\tilde{\mathrm{b}}_{\mathrm{j}}$ & $\cdots$ & $\tilde{\mathrm{b}}_{\mathrm{n}}$ & \\
\hline
\end{tabular}


TABLE III: Fuzzy transportation problem with fuzzy cost

\begin{tabular}{|c|c|c|c|c|}
\hline & $\mathrm{D}_{1}$ & $\mathrm{D}_{2}$ & $\mathrm{D}_{3}$ & Supply $\left(\tilde{\mathrm{a}}_{\mathrm{i}}\right)$ \\
\hline $\mathrm{S}_{1}$ & $(4,9,3,10)$ & $(2,5,1,4)$ & $(5,8,3,10)$ & $(5,7,4,2)$ \\
\hline $\mathrm{S}_{2}$ & $(9,12,1,14)$ & $(5,8,2,4)$ & $(9,13,2,15)$ & $(7,8,3,2)$ \\
\hline $\mathrm{S}_{3}$ & $(12,20,1,7)$ & $(5,10,0,5)$ & $(5,8,1,3)$ & $(5,8,1,3)$ \\
\hline $\operatorname{Demand}\left(\tilde{\mathrm{b}}_{\mathrm{j}}\right)$ & $(5,8,2,4)$ & $(8,9,4,1)$ & $(4,6,2,2)$ & \\
\hline
\end{tabular}

Table IV: Comparison of Optimal fuzzy transportation cost

\begin{tabular}{|l|l|l|l|l|}
\hline \multirow{2}{*}{$\begin{array}{l}\text { Fuzzy } \\
\text { transportation } \\
\text { models }\end{array}$} & \multicolumn{4}{|l|}{ Optimal fuzzy transportation cost } \\
\cline { 2 - 5 } & $\begin{array}{l}\mathrm{L}(\mathrm{x})=\mathrm{R}(\mathrm{x}) \\
=\max \{0,1-|\mathrm{x}|\}\end{array}$ & $\begin{array}{l}\mathrm{L}(\mathrm{x})=\mathrm{R}(\mathrm{x}) \\
=\mathrm{e}^{-\mathrm{x}}\end{array}$ & $\begin{array}{l}\mathrm{L}(\mathrm{x})=\max \{0,1-|\mathrm{x}|\} \\
\text { and } \mathrm{R}(\mathrm{x})=\mathrm{e}^{-\mathrm{x}}\end{array}$ & $\begin{array}{l}\mathrm{L}(\mathrm{x})=\mathrm{e}^{-\mathrm{x}} \text { and } \\
\mathrm{R}(\mathrm{x})=\max \{0,1-|\mathrm{x}|\}\end{array}$ \\
\hline Equality Model & $\begin{array}{l}(84.89,147.88,36.50, \\
104.32)\end{array}$ & $(92.5,161,39.5,118)$ & $(102.75,178.75,45.25,131)$ & $(85.5,147.25,37.25,108)$ \\
\hline Inequality Model & $\begin{array}{l}(90.75,163.25,39.75, \\
117)\end{array}$ & $(92.5,161,39.5,118)$ & $(102.75,178.75,45.25,131)$ & $(85.5,147.25,37.25,108)$ \\
\hline
\end{tabular}

\section{REFERENCES}

[1] Freguson, A.R., and Dantzig, G.B. 1995. Theallocations of aircraft to routes - An example

[2] Charnes, A., and Cooper, W.W. 1961.Management models and industrialapplications of linear programming, Vols. Iand II, Wiley, New York.

[3] Garvin, W.W. 1960. Introduction to linearprogramming. Mc Graw-Hill. New York.

[4] Hadley, G. 1962. Linear programming,Addison-Wesley, reading, mass.

[5] Balachandran, V., and Thompson, G.L. 1975.An Operator theory of parametric programming for the generalizedtransportation problem: I basic theory, Nav.Res. Log. Quart., 22, 79-100.

[6] Balachandran, V., and Thompson, G.L. 1975.An Operator theory of parametric Programming for the generalizedtransportation problem: II Rim, cost andbound operators, Nav. Res. Log.Quart., 22,101-125.

[7] Balachandran, V., and Thompson, G.L. 1975.An Operator theory of parametric programming for thegeneralized transportation problem: III Weight operators,Nav. Res. Log. Quart., 22,297-315.

[8] Hughes, J.B. 1987. A multiobjective cuttingstock problem with stochastic demand in theAluminum industry, presented at the secondwork shop on mathematics in industry, ICTP,Trieste, Itlay. of linear programming under uncertaindemand. Management science. $3,45-73$.

[9] Ignizio, J.P., and Cavalier, T.M. 1994. Linearprogramming, Prentice-Hall, EnglewoodCliffs, NJ.

[10] Stroup, J.S., and Wollmer, R.D. 1992. Afuel management model for the airlineindustry, Journal of operations Research Society of America, 40, 229-237.

[11] Balas, E. 1966. The dual method for the generalized transportation problem, Management Science, 12(7), 555-568.

[12] Bit, A.K., Biswal, M.P., and Alam, S.S.1992. Fuzzy programming approach to multicriteria decision making transportationproblem. Fuzzy Sets and Systems, 50,135141.

[13] Chanas, S., and Kuchta, D. 1996. Aconcept of the optimal solution of the transportation problem with fuzzy costcoefficients. Fuzzy Sets and Systems 82(3),299-305.

[14] Hussien, M.L. 1998. Complete solutions of multiple objective transportation problem with possibilistic coefficients. Fuzzy Sets and Systems 93(3),293-299.

[15] Li, L., and Lai, K.K. 2000. A fuzzyapproach to the multi objective transportation problem. Computers and Operations Research 27, 43-57. 
[16] Zimmermann, H.J. 1978. Fuzzyprogramming and linear programming withseveral objective functions, Fuzzy SetsSyst. $1,45-55$.

[17] Oheigeartaigh, M. 1982. A fuzzytransportation algorithm, Fuzzy Sets Syst. 8,235-24 3.

[18] Chanas, S., Kolodziejckzy, W., and Machaj, A.A. 1984. A fuzzy approach to the transportation problem, Fuzzy Sets andSystems. 13, 211-221.

[19] Chanas, S., and Kuchta, D. 1996. A Concept of the optimal solution of the transportation problem with fuzzy cost coefficients, Fuzzy Sets and Systems. 82 , 299-305.

[20] Saad, O.M., and Abbas, S.A. 2003. A parametric study on transportation problem under fuzzy environment, Journal of Fuzzy Mathematics. 11, 115-124.
[21] Liu, S.T., and Kao, C. 2004. Solving fuzzy transportation problems based on extension principle, European Journal of OperationalResearch. 153, 661-674.

[22] Dubois, D., and Prade, H. 1980. Fuzzy sets and systems: theory and applications. Academic Press, New York.

[23] Yager, R. R. 1981. A procedure for ordering fuzzy subsets of the unit interval. Information $\backslash$ Sciences 24 , 143-161.

[24] Kaur, A., and Kumar, A. 2011. A new method for solving fuzzy transportation problems using ranking function, Applied Mathematical Modelling 35, 56525661 